\title{
Correction to: DNA damage response and repair pathway modulation by non-histone protein methylation: implications in neurodegeneration
}

\author{
Madhusoodanan Urulangodi ${ }^{1}$ (D) Abhishek Mohanty ${ }^{2}$ \\ Published online: 29 September 2020 \\ (C) The International CCN Society 2020
}

Correction to: Journal of Cell Communication and Signaling (2020) 14: 31-45 https://doi.org/10.1007/s12079-019-00538-2

The original version of this article unfortunately requires correction where the name of a methyltransferase was represented wrongly. On page no 9, under Lysine methylation of mitochondrial proteins section. The author stated that "Recently, FAM173B was shown to methylate another mitochondrial enzyme, Adenine nucleotide translocase (ANT) (Malecki et al. 2019)". It is actually FAM173A and not FAM173B and therefore, the sentence may be modified to "Recently, FAM173A was shown to methylate a mitochondrial enzyme, Adenine nucleotide translocase (ANT) (Malecki et al. 2019)". It is correctly represented as FAM173A in Table 2. The correction is shown below:
Update: "Recently, FAM173B was shown to methylate another mitochondrial enzyme, Adenine nucleotide translocase (ANT) (Malecki et al. 2019)".

To: "Recently, FAM173A was shown to methylate a mitochondrial enzyme, Adenine nucleotide translocase (ANT) (Malecki et al. 2019)".

Publisher's note Springer Nature remains neutral with regard to jurisdictional claims in published maps and institutional affiliations.

The online version of the original article can be found at https:/doi.org/ 10.1007/s12079-019-00538-2

Madhusoodanan Urulangodi drmadhusoodanan@sctimst.ac.in

Abhishek Mohanty abhishek.m.iisc@gmail.com

1 Department of Biochemistry, Sree Chitra Tirunal Institute for Medical Sciences and Technology,

Thiruvananthapuram, Kerala PIN-695011, India

2 Rajiv Gandhi Cancer Institute and Research Centre, New Delhi PIN-110085, India 